EPiC Series in Engineering
Volume 3, 2018, Pages 555-563
HIC 2018. 13th International
Conference on Hydroinformatics

\title{
Simulation architecture based on distributive MDP for inland waterway management
}

\author{
G. Desquesnes ${ }^{1 *}$, D. Alves ${ }^{1}$, E. Duviella ${ }^{1}$, G. Lozenguez ${ }^{1}$, A. Doniec ${ }^{1}$ \\ ${ }^{1}$ Informatics and Automatics Department, IMT Lille Douai, Lille, France \\ \{guillaume.desquesnes, Debora.alves, eric.duviella, \\ guillaume.lozenguez, arnaud.doniec\}@imt-lille-douai.fr
}

\begin{abstract}
Climate change should affect inland waterways in a close future. The study of their resilience against climate change requires an optimal resource allocation. Indeed, it is possible to analyze the global change effects on inland waterways only if the water resources allocation is optimal. In addition, the events due to climate change are not deterministic. It is obvious that it is not possible to predict precisely their occurrence time, their magnitude and their duration. Hence, it is necessary to consider uncertainties in climate projections, and more precisely uncertainty bounds on these predictions. The objective of this paper is to propose a simulation architecture of inland waterways that couples simulation software of their dynamics and an optimal water resources allocation approach under uncertainties based on Markov Decision Process. The proposed simulation architecture and the designed tools are detailed and implemented by considering some expected climatic events on a part of the inland waterways in the north of France.
\end{abstract}

\section{Introduction}

The study of climate change has been achieved in the framework of several projects around the world since several years. The IPCC in their report states that climate change will have impacts on temperature and rainfall in near and far future (IPCC, 2014). The climate change impacts on rivers and available water resource have been studied at the European area in Glowa ${ }^{\dagger}$, Kliwas $^{\ddagger}$, Ecconet $^{\S}, \mathrm{R} 2 \mathrm{D} 2^{* *}$, Explore2070 ${ }^{\dagger \dagger}$ projects. In (Beuthe et al, 2014), a methodology based on the NODUS transport model

\footnotetext{
${ }^{*}$ Corresponding author

$\dagger$ http://www.glowa.org/

$\$$ http://www.kliwas.de

$\S$ http://www.ecconet.eu/deliverables/index.htm

$* *$ https://r2d2-2050.cemagref.fr/

${ }^{\dagger \dagger}$ http://www.developpement-durable.gouv.fr/Evaluation-des-strategies-d.html
} 
has been proposed to estimate the climate change impacts on transport on the Rhine and Danube corridors. The conclusion of this study is that impacts will come more from a new multimodal transport split than climate change over the period 2020-2050. More recently, a study of the climate change impacts on the inland waterways of the north of France has been proposed (Gepet-eau project $\$$ ). An integrated volume model and a dynamic directed flow graph have been designed to model the inland waterways (Nouasse et al, 2015). Based on these tools, optimal resources allocation planning can be performed according to Constraint Satisfaction Problems (CSP) (Nouasse et al, 2016), or Quadratic Optimization Problems (Duviella et al, 2016). Software has been developed to study the resilience of waterways against extreme climate events and the increase of the navigation demand (Duviella et al, 2017). A user's web-interface allows modelling several configurations of waterways and specifying scenarios. The optimal water resources allocation planning is then determined according to a CSP over a one-step horizon. This approach has been used to study the resilience of a part of the inland waterways of the north of France. However, the proposed approach is determinists. That means that none uncertainty has been taken into account. It is a priori the main drawback of these proposed tools particularly in a context of climate change where forecasts are intrinsically uncertain. To overcome this issue, an optimal water resources allocation planning based on Markov Decision Process (MDP) has been proposed in (Desquesnes et al, 2016) then extended in (Desquesnes et al, 2017) by introducing distributed properties that are required for large systems. Distributed MDP are dedicated to large stochastic dynamic systems and therefore are well adapted to the study of the climate change impacts on inland waterways. The objective of this paper is to present a simulation architecture that links simulation software able to reproduce the dynamics of inland waterways and the designed distributed MDP method. This simulation architecture can be used to simulate some expected climatic events on inland waterways. It is described in section 2 . Section 3 is dedicated to the presentation of the distributed MDP. A part of the inland waterways of the north of France is presented in section 4, and used to illustrate the benefit of the simulation architecture by considering several climate events.

\section{Simulation architecture}

\subsection{Integrated model of inland waterways}

Inland waterways are composed of several Navigation Reaches (NR) that are interconnected and principally linked by locks (see Figure 1.a). They compose a large network which configurations can be modelled by considering three elementary links: the linear link, the tributary and the distributary. With the aim to well understand the water volume dispatching among the network, an integrated model is proposed and depicted in Figure 1.b.

This deterministic model aims at reproducing the dynamics of the network by computing the water volume balance in each $\mathrm{NR}_{\mathrm{i}}$ at each step time $\mathrm{k}$, such as:

$$
V_{i}(k)=V_{i}(k-1)+V_{i}^{s, c}(k)-V_{i}^{e, c}(k)+V_{i}^{c}(k)+V_{i}^{s, p}(k)-V_{i}^{e, p}(k)+V_{i}^{u}(k)+V_{i}^{g, u}(k)
$$

with $\mathrm{V}_{\mathrm{i}}(\mathrm{k})$ the water volume in the $\mathrm{NR}_{\mathrm{i}}$ at time $\mathrm{k}$, and:

- $\quad V_{i}^{s, c}$ the controlled volumes from all the upstream NR that supply the NRi (s: supply, c: controlled),

- $\quad V_{i}^{e, c}$ the controlled volumes from the $\mathrm{NR}_{\mathrm{i}}$ that empty it (e: empty),

- $\quad V_{i}^{c}$ the controlled volumes from water intakes that can supply or empty the $\mathrm{NR}_{\mathrm{i}}$,

- $\quad V_{i}^{s, p}$ the controlled volumes from pump that can supply the $\mathrm{NR}_{\mathrm{i}}$ (p: pumped),

\footnotetext{
\$ https://gepeteau.wordpress.com/
} 
- $\quad V_{i}^{e, p}$ the controlled volumes from pump that can empty the $\mathrm{NR}$,

- $\quad V_{i}^{u}$ the uncontrolled volumes from natural rivers, rainfall-runoff, etc. (u: uncontrolled),

- $\quad V_{i}^{g, u}$ the uncontrolled volumes from exchanges with groundwater (g: groundwater), where $V_{i}^{c}, V_{i}^{u}$ and $V_{i}^{g, u}$ are signed; positive if the $\mathrm{NR}_{\mathrm{i}}$ is supplied, negative otherwise.

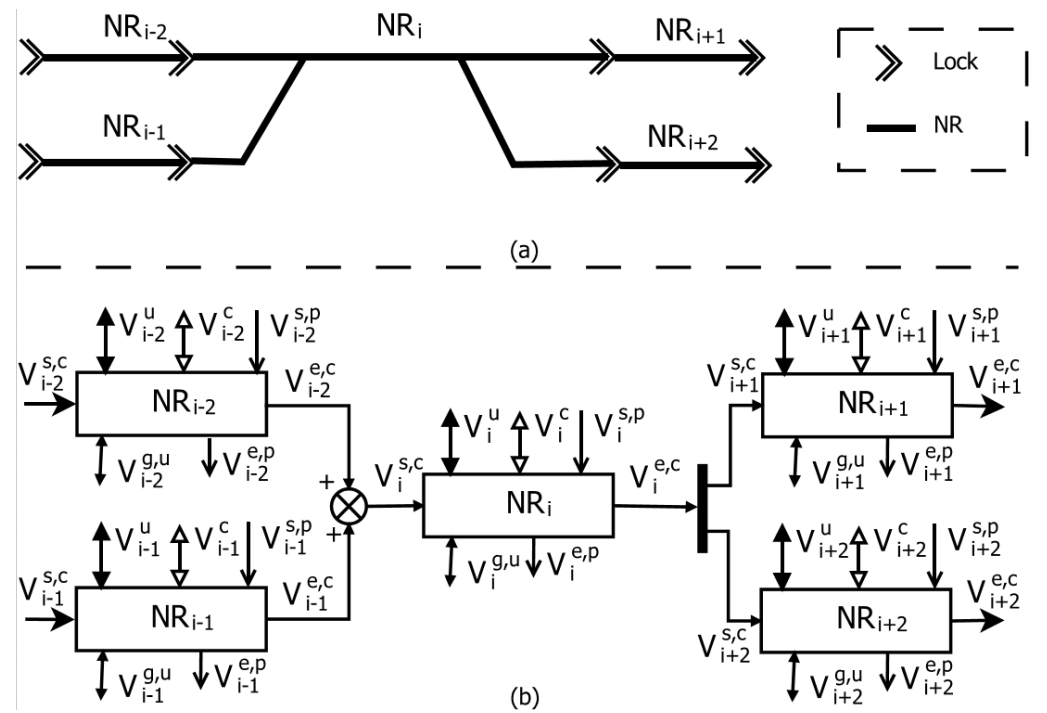

Figure 1: (a) Inland navigation network, (b) Integrated model

The main objective of the inland waterway managers is to keep the water level of each NR close to the Normal Navigation Level (NNL) and inside the navigation rectangle. The navigation rectangle is defined with two boundaries; the High Navigation Level (HNL) and the Low Navigation Level (LNL). It leads to the definition of the objective volume $V_{i}^{N N L}$ and the interval around the current volume such as:

$$
V_{i}^{L N L} \leq V_{i}(k) \leq V_{i}^{H N L}
$$

In the same way, each controlled volumes depend on the characteristics of hydraulic structures. They are bounded by:

$$
\left\{\begin{array}{l}
\frac{V_{i}^{s, c}}{\overline{V_{i}^{e, c}}} \leq V_{i}^{s, c} \leq \overline{V_{i}^{s, c}} \\
\frac{V_{i}^{c}}{V_{i}^{c}} \leq \overline{V_{i}^{e, c}} \\
\underline{V_{i}^{s, p}} \leq \overline{V_{i}^{c}} \\
\overline{V_{i}^{e, p}} \leq V_{i}^{s, p} \leq \overline{V_{i}^{s, p}} \\
. \overline{V_{i}^{e, p}}
\end{array}\right.
$$

with $\underline{V_{i}}$ and $\overline{V_{i}}$ the inferior and superior bounds respectively. For more details, please refer to (Nouasse et al, 2016).

Based on these relations, a stochastic integrated model is proposed to deal with the uncertainties related to the uncontrolled volumes and the navigation demand such as example. Relation (1) becomes: 


$$
\begin{aligned}
& \quad V_{i}(k)=V_{i}(k-1)+\left(V_{i}^{s, c}(k)+\Delta_{V_{i}^{s, c}}(k)\right)-\left(V_{i}^{e, c}(k)+\Delta_{V_{i}^{e, c}}(k)\right)+ \\
& \left(V_{i}^{c}(k)+\Delta_{V_{i}^{c}}(k)\right)+\left(V_{i}^{s, p}(k)+\Delta_{V_{i}^{s, p}}(k)\right)-\left(V_{i}^{e, p}(k)+\Delta_{V_{i}^{e, p}}(k)\right)+\left(V_{i}^{u}(k)++\Delta_{V_{i}^{u}}(k)\right)+ \\
& \left(V_{i}^{g, u}(k)+\Delta_{V_{i}^{g, u}}(k)\right)
\end{aligned}
$$

with $\Delta_{V_{i}^{i}}(k)$ the uncertainties that is related to the known controlled water volumes and estimated uncontrolled ones. It is supposed that uncertainties follow Normal distributions. The uncertainties related to uncontrolled water volumes are more important than those related to controlled water volumes. Moreover, they are highly dependent of climate events. As an example during rainy events, uncontrolled volumes from natural rivers depend directly to the intensity of the rain. This model is implemented with Matlab/Simulink.

\subsection{Management objectives}

It appears now obvious that the main management objective consists in dispatching the available water resource among the inland navigation network to guarantee the navigation conditions (see relation 2). Hence, a predictive water allocation planning over a future horizon of several days has to be designed. This planning method has to deal with strong uncertainties. Hence, a distributed MDP has been designed to reach these objectives. Distributed MDP are dedicated to large stochastic dynamic systems.

The water allocation planning is performed according to the controlled structures such as controlled gates to dispatch the water volumes. It respects the constraints on these structures (see relation 3). Moreover, it has to respect the configuration of the inland navigation network and the dispatching time. Indeed, it is not possible to supply instantaneously a NR that is located downstream the navigation network with water coming from a NR upstream when they are separated by several NR. However, due to the sample time that is considered, i.e. several hours that corresponds to the navigation scheduling time, the transfer of water volumes between two neighbored NRs is possible between two sample time.

\subsection{Simulation architecture}

The objective of the simulation architecture is to implement and to test the designed distributed MDP algorithm on a simulator of inland waterways. The architecture is depicted in Figure 2.

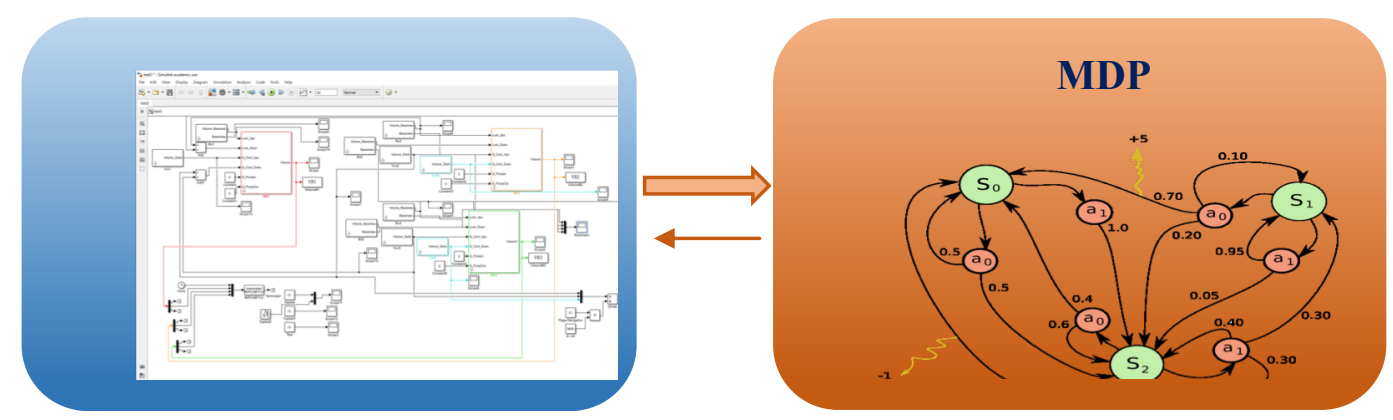

Figure 2: Simulation architecture based on distributed MDP for inland waterway management

The simulator of inland waterways is realized according to Matlab/Simulink that offers a powerful simulation tool. It is possible to reproduce the dynamics of each NR, to link these reaches between them according to the waterways configuration, to implement the dynamics of the gates and locks, to consider uncertainties in the simulation, and finally to tune the step time and the time of the simulation. Each NR 
is modelled with a Simulink block as depicted in Figure 3. It allows considering as inputs the volumes due to lock operations upstream and downstream, the controlled discharges that are used to compute the volume on the considered time and uncontrolled discharges with uncertainties. The output of the block is the volume of the NR at each step time $\mathrm{k}$. All NR blocks are then linked together following the network configuration.

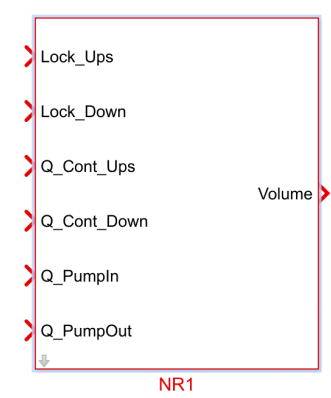

Figure 3: Simulink block of one NR

The distributed MDP algorithm is implemented in Java and is run on multiple machines. A communication link between Matlab/Simulink and the distributed MDP algorithm has been built to exchange data between these two environments. Characteristics of the networks (number of reaches, size of the reaches, volume of the lock chambers, etc.) and information about the simulated scenarios (uncontrolled inputs that are modelled with Normal distributions, climatic events, etc.) are sent to the Java environment where distributed MDP solver provides locally optimal policies. Then, these policies are used to allocate setpoints to each controlled gates at each time step.

Hence, when a simulated scenario is defined over a future horizon $\mathrm{H}$ in the Matlab environment, sent to Simulink and to the distributed MDP solver, once a solution of optimal planning is found, the simulation can start. At each time step, Simulink exchanges information on the current state of the modelled waterways with the distributed MDP solution to retrieve the setpoint for each controlled gate. These setpoints are ranked in a table for each step time according to the possible states of the water resource. These possible states are determined according to the bounded uncertainties on all the considered variables. Thus, new setpoints derive from the table.

\section{Distributed MDP}

Markov Decision Process (MDP) is a tool for modelling control possibility of a discrete system. Planning algorithms, such as Value-iteration, applied to MDP produce a mapping of control to each possible state of the system. MDPs are powerful but lack the scalability to model realistic systems. Distributing the model over multiple agents allow to alleviate this limitation. In the proposed approach, each agent controls a subset of gates and locks and aims to locally optimize the water level of NRs it affects with respect to the uncertainties of traffic and flow. Connections are made between agents when multiple agents affect a same reach. Two agents affecting a same reach are considered neighbors. A distributed algorithm, see algorithm 1, has been introduced in (Desquesnes et al, 2017). It consists of iteratively improving the local policy of agents using offline coordination. At each iteration, agents start by exchanging the most information possible about their local policy to their neighbors. The exchanged is limited to the common knowledge of both agents, i.e. NRs affected by both agents as well as the controlled locks and gates affecting them. This increases the knowledge of agents about their neighbor choices without requiring complete information of their local models. Using the received policies from its neighborhood, each agent will be able to update its model and compute a new local policy. 
Conflicting policy changes are avoided by allowing at most one agent per neighborhood to update its local policy during a single iteration. The agent who keeps its policy at a given iteration corresponds to the agent having the highest improvements of policy in the new models in its neighborhood, with respect to an exploration heuristic. Thus the algorithm automatically usually converges toward an acceptable. In some cases, the algorithm may fall into cycles. Those can be detected when a series of improvement policy values and thus can be broken by preventing agents to update local policies while cycling. The algorithm stops when no agent is able to improve its policy. This is detected locally by using counters as presented in Distributed Breakout Algorithm.

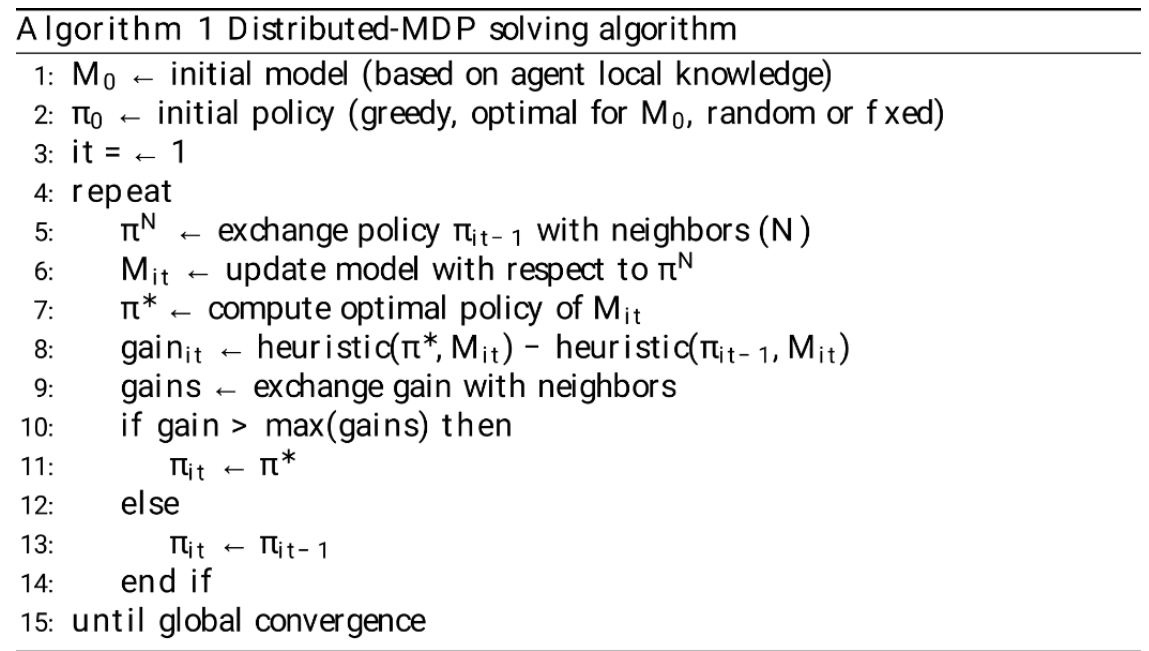

The proposed simulation architecture is used for the management of a real inland waterways located in the north of France. Some scenarios are simulated to highlight the performance of the approach..

\section{Inland waterways in a climate change context}

A part of the inland waterways in the north of France is considered to illustrate the benefit of the simulation architecture by simulating several climate events. It is composed of three interconnected reaches from the city of Douai to the cities of Arques and Lille in the north of France (see Figure 4). The characteristics of each NR are given in Table 1.

\begin{tabular}{ccccccc}
\hline NR & Name & L $[\mathrm{km}]$ & $1[\mathrm{~m}]$ & NNL $(\mathrm{m})$ & LNL $(\mathrm{m})$ & HNL $(\mathrm{m})$ \\
\hline 1 & Douai-Don-Cuinchy & 56.724 & 41.8 & 3.7 & -0.05 & +0.1 \\
2 & Cuinchy-Fontinettes & 42.3 & 52 & 4.3 & -0.05 & +0.05 \\
3 & Don-Grand-Carré & 25.694 & 45.1 & 3.3 & -0.05 & +0.05 \\
\hline
\end{tabular}

Table 1: NR properties, with L the length, 1 the width, LNL and HNL are relative to NNL

The range of the controlled discharges from rivers, i.e. $Q_{i}^{c}$, the estimated mean values of the uncontrolled discharges, i.e. $Q_{i}^{u}$, the size of the lock chamber and the operating ranges of the controlled gates between NR are given in Table 2. Based on these discharges and the considered period of time, the corresponding volumes are easily computed. 


\begin{tabular}{ccccccc}
\hline $\mathrm{NR}$ & $Q_{i}^{c}\left[\mathrm{~m}^{3} / \mathrm{s}\right]$ & $Q_{i}^{u}\left[\mathrm{~m}^{3} / \mathrm{s}\right]$ & $Q_{i}^{S, c}\left[\mathrm{~m}^{3} / \mathrm{s}\right]$ & $Q_{i}^{e, c}\left[\mathrm{~m}^{3} / \mathrm{s}\right]$ & $\begin{array}{c}\text { chamber up. } \\
{\left[\mathrm{m}^{3}\right]}\end{array}$ & $\begin{array}{c}\text { chamber dw. } \\
{\left[\mathrm{m}^{3}\right]}\end{array}$ \\
\hline 1 & {$[-1 ;-1]$} & 6.56 & - & - & 6,709 & - \\
2 & - & 0.63 & {$[0 ; 6.4]$} & - & 3,526 & 23,000 \\
3 & - & 1.2 & {$[0 ; 30]$} & {$[0 ; 60]$} & 5,904 & 7,339 \\
\hline
\end{tabular}

Table 2: Hydraulic structures properties, with chamber up (resp. $\mathrm{dw}$ ) is the exchanged volume at each lock operation

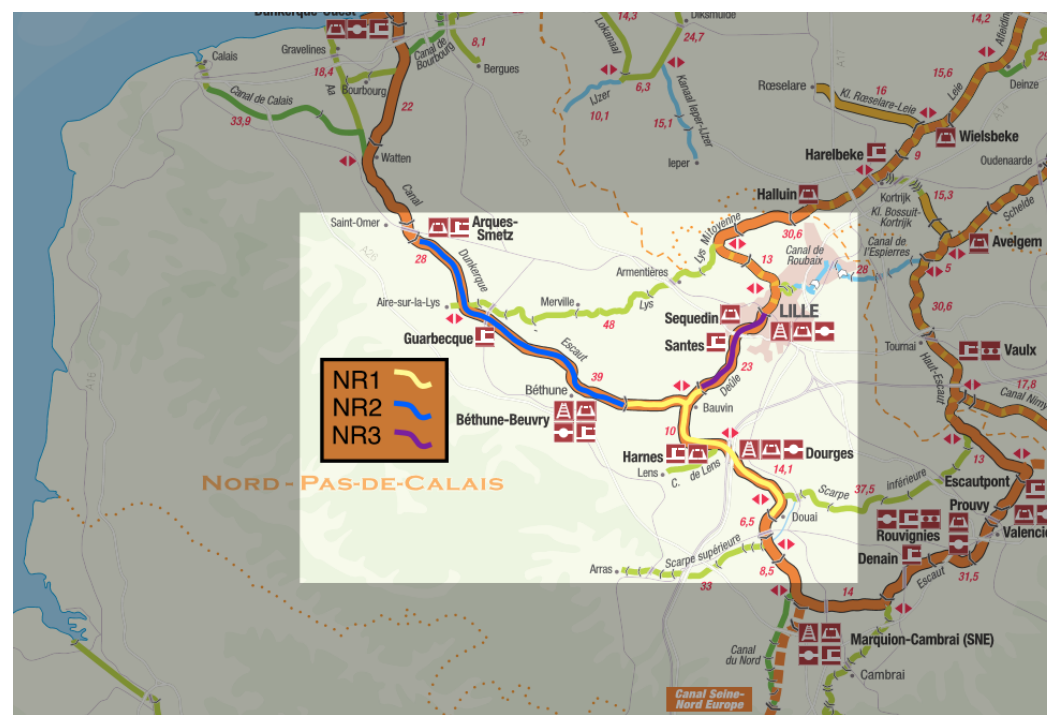

Figure 4: Section of the inland waterways in the north of France

A Simulink model of the studied network has been designed. The navigation scheduled time is tuned at 12 hours. That means that half of the day, the navigation is stopped (during the night). The average number of lock operations are given in Table 3. These numbers are used to design the scenarios.

\begin{tabular}{cccccc}
\hline Lock & $\begin{array}{c}\text { Douai } \\
\left(\mathrm{NR}_{1} \text { up }\right)\end{array}$ & $\begin{array}{c}\text { Cuinchy } \\
\left(\mathrm{NR}_{2} \text { up }\right)\end{array}$ & $\begin{array}{c}\text { Don } \\
\left(\mathrm{NR}_{3} \text { up }\right)\end{array}$ & $\begin{array}{c}\text { Fontinettes } \\
\left(\mathrm{NR}_{2} \mathrm{dw}\right)\end{array}$ & $\begin{array}{c}\text { Grand Carré } \\
\left(\mathrm{NR}_{3} \mathrm{dw}\right)\end{array}$ \\
\hline Number & 21 & 13 & 14 & 10 & 16 \\
\hline
\end{tabular}

Table 3: Average daily number of lock operations

The predictive horizon $\mathrm{H}$ is tuned to 4 days corresponding to 8 sample times. A first scenario is based on the average daily number of lock operations and the uncontrolled discharges without uncertainties over 4 days. The objective is to show the performance of the distributed MDP approach in a deterministic case. Results are given in Figure 5 for the three NRs where the levels are depicted in relative according to the NNL and the boundaries are normalized in percentages. It can be observed that the navigation objectives are guaranteed for the three NRs. The simulation shows that the approach is unable to maintain exactly the NNL at all time, due to the discretization required by the MDP modelling. It induces uncertainties about the current state of the systems and its modifications.

The second scenario aims at simulating the effects of rainy events of strong intensity. The main consequences are observed on the uncontrolled discharges $Q_{i}^{u}$ of the three NRs. Indeed, the rain events will lead to increase the average values of uncontrolled discharges of all NRs by $150 \%$ between time 3 and 5, then 6 and 8. Even if the distributed MDP has no prior knowledge of these events, the levels of the three NRs are kept in the navigation rectangle as it is shown in Figure 6. This scenario confirms the ability of the approach to deal with strong uncertainties. 


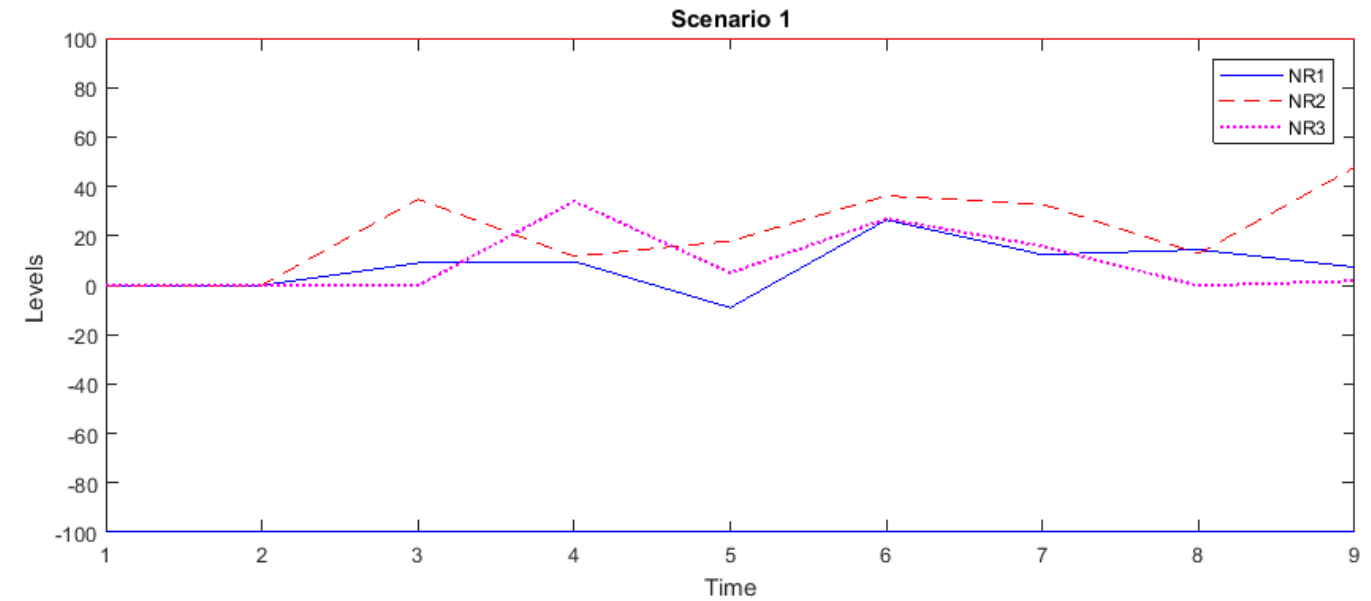

Figure 5: Scenario 1 with level of $\mathrm{NR}_{1}$ depicted in blue continuous line, of $\mathrm{NR}_{2}$ in red dashed line and of $\mathrm{NR}_{3}$ in magenta dotted line

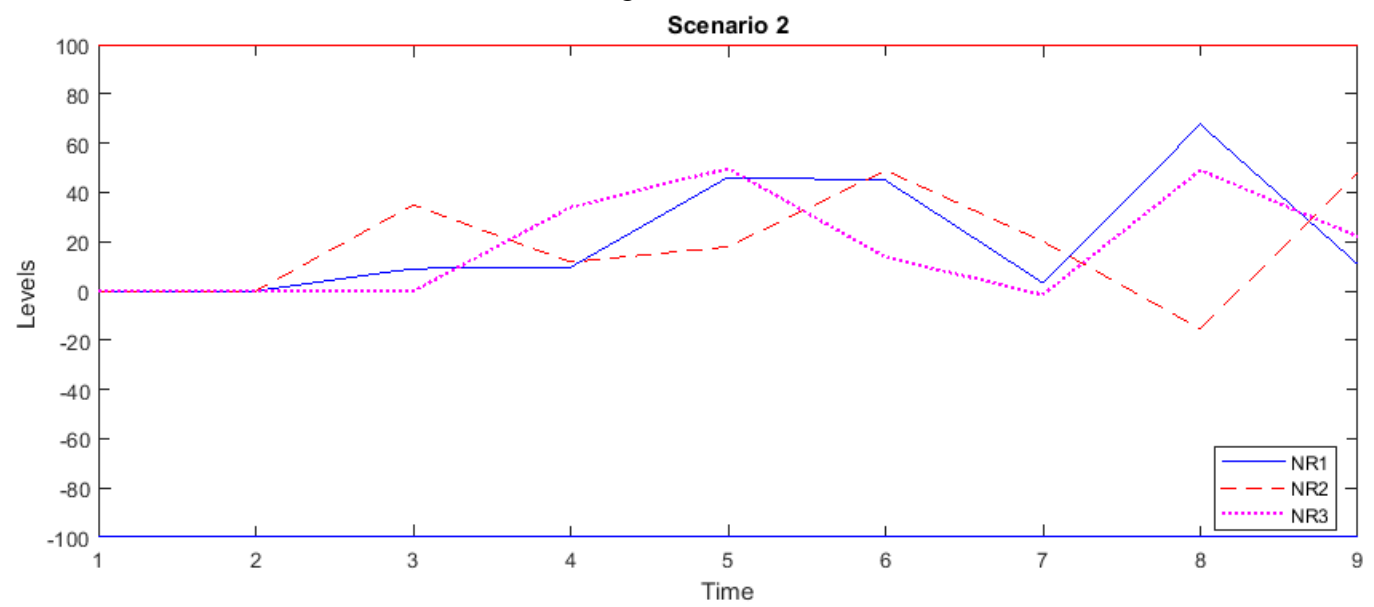

Figure 6: Scenario 2 with level of $\mathrm{NR}_{1}$ depicted in blue continuous line, of $\mathrm{NR}_{2}$ in red dashed line and of $\mathrm{NR}_{3}$ in magenta dotted line

The proposed simulation architecture makes easy the simulation of several scenarios. The management horizons, the uncertainties, the navigation demand, the events like drought or rainy periods, etc., can be easily modified. Thus, situations with normal conditions, drought and rain events can be simulated by considering uncertainties. The distributed MDP solver provides solutions by anticipating the impacts of climatic events, and therefore guaranteeing the navigation conditions.

\section{Conclusion}

In this paper, a simulation architecture that is based on simulation software of the inland waterways dynamics and an optimal water resources allocation approach under uncertainties is proposed. The dynamics of inland waterways are simulated thanks to the software Matlab/Simulink that offers a good 
interface to build several scenarios. It is easily possible to modify the configuration of the networks, the operating range of the controlled gates, the uncertainties linked to the navigation demand or uncontrolled inputs coming from rain events. Once the scenario is built, all the data are sent to a distributed MDP solver that provides locally optimal policies by considering a management horizon of several days. These policies are then used to allocate setpoints to each controlled gates at each time step. According to these setpoints, it is possible to guaranty the navigation condition by anticipating the impacts of some events dealing with uncertainties. A part of the inland waterways in the north of France is presented to illustrate the proposed simulation approach. Two scenarios, one without uncertainties, the second with strong uncertainties coming from non-expected rainy events, demonstrate the performance of the designed approach. Future works consist in considering more scenarios, in studying the resilience of inland waterways and in proposing some relaxation possibilities according to structural solutions such as pump implementation or flood expending zones.

\section{References}

IPCC (2014), Climate Change 2014, The Core Writing Team, R. K. Pachauri and L. Meyer, Synthesis Report, https://www.ipcc.ch/pdf/assessment-report/ar5/syr/SYR \AR5 _FINAL \full.pdf.

Beuthe M., B. Jourquin, N. Urbain, I. Lingemann, B. Ubbels (2014), Climate change impacts on transport on the Rhine and Danube: A multimodal approach. Transportation Research Part D: Transport and Environment, vol 27, pp 6-11.

Nouasse H., L. Rajaoarisoa, A. Doniec, P. Chiron, E. Duviella, B. Archimède, K. Chuquet (2015), Study of drought impact on inland navigation systems based on a flow network model, ICAT, Sarajevo, Bosnia Herzegovina, 29-31 October.

Nouasse H., A. Doniec, G. Lozenguez, E. Duviella, P. Chiron, B. Archimède, K. Chuquet (2016), Constraint satisfaction problem based on flow transport graph to study the resilience of inland navigation networks in a climate change context, IFAC Conference on Manufacturing Modelling, Management, and Control, MIM, Troyes, France, 28-30 June.

Duviella E., H. Nouasse, A. Doniec, K. Chuquet (2016), Dynamic Optimization Approaches for Resource Allocation Planning in Inland Navigation Networks, ETFA'2016, Berlin, Germany, 6-9 September.

Duviella E., A. Doniec, H. Nouasse, F. Tempet (2017), Constraint satisfaction problem vs quadratic optimization method to deal with the effect of climate change on inland waterways, 37th IAHR World Congress, Kuala Lumpur, Malaysia, 13-18 August.

Desquesnes G., G. Lozenguez, A. Doniec, E. Duviella (2016), Dealing with Large MDPs, case study of waterway networks supervision, Advances in Distributed Computing and Artificial Intelligence Journal, Volume 5, Issue 4, pp 71-84.

Desquesnes G., G. Lozenguez, A. Doniec, E. Duviella (2017), Distributed MDP for water resources planning and management in inland waterway, IFAC WC, Toulouse, France, 9-14 July. 